\title{
Evaluation of Niche Suitability of Regional Technological Innovation Ecosystem \\ Wei Chen
}

Xi'an International University, Xi'an, China

\begin{abstract}
Keywords: Regional Technological Innovation, Ecosystem, Niche Suitability
\end{abstract}
\begin{abstract}
Analysis of Regional Technological Innovation Ecosystem Suitability from the Perspective of Economic and Technological Environment. The evaluation index system of regional ecosystem of technical innovation ecosystem was constructed and the niche evaluation model was selected to estimate the suitability of regional ecosystems. The empirical results show that the technological innovation ecosystem in eastern China is more suitable, the western region is lower and the central region is centered.
\end{abstract}

\section{Introduction}

With the economic transformation, Chinese economic development has been driven by factors to innovation, innovation, entrepreneurship and emerging industries as the core capacity of the innovative cluster to become a regional competitiveness of the concentrated expression of the party's Eighth Plenary Session to further emphasize the adhere to innovation and development, innovation will be placed in the core of the overall development of the country. The innovation effect is influenced by the system environment. In the innovative ecosystem, the innovation system and the innovation environment, through the technology, talent, capital, information mutual influence, mutual restraint, form a collaborative evolution, innovation and opening up the complex system. The construction of regional innovation ecosystem and the development of regional innovation ability is an important way to enhance the national competitiveness, to explore the regional evolution of ecosystems within the main body of collaborative evolution law, to achieve regional synergistic development, become the theoretical issues need to study and practical difficulties. Evolutionary ideology and niche suitability provide a new perspective for the study of regional synergistic evolution mechanism of innovative ecosystems.

\section{Construction of Evaluation System of Regional Technological Innovation Ecosystem Suitability}

Regional technological innovation Ecosystem is an organic whole that interacts and interacts with each other within a certain area, innovation community and innovative environment. The suitability of the technological innovation ecosystem in this paper is to analyze the degree of profitability of the innovation subject in the region from the perspective of regional economic and technological environment. Its evaluation is composed of multiple parts, mainly covering the innovation group, the economic environment, Innovative resources and technical environment and so on. Innovative groups are mainly composed of enterprises, scientific research institutions and institutions of higher learning. Enterprises are the main innovation, in which industrial enterprises in the innovation group in the main position. Economic environment includes macroeconomic environment, micro-market environment, cooperation and exchange environment and information environment and other components. Innovative resources are mainly people, financial, material and other aspects. Technical environment is including the generation of innovative technology, circulation and application and so on.

The evaluation index system of regional technical innovation ecosystem suitability should be a scientific, standardized, systematic and standardized system, which can comprehensively and objectively reflect the whole picture of regional technological innovation ecosystem, so as to carry 
out the suitability of regional technological innovation ecosystem objective comment. The establishment of the evaluation index system should meet the following objectives:

It clearly reflects the basic situation of regional ecosystems' suitability is fully utilizes quantitative indicators to understand the current situation and development trend of regional technological innovation ecosystem suitability, and comprehensively evaluate the level of regional ecosystems for the development of efficient ecosystems. Innovation strategy is to provide scientific basis.

Through the analysis of the relevant indicators of the regional technological innovation ecosystem suitability, it helps the decision-makers to control the overall situation, grasp the advantages and disadvantages of the regional innovation ecological environment, and make the regional innovation science and technology policy service better.

Around the above objectives, in the design of regional technological innovation ecosystem suitability index system in the process should follow the following principles:

Systematic principle. The evaluation index of the suitability of the ecosystem is suitable for the prediction and measurement of the subject and the environment of the innovation. The scope and problems involved are extensive and complex and the factors of each aspect should be considered in the system design. Fully consider the relevance of the indicators, integrity and objectives, to ensure that the set of indicators of the system is complete and orderly.

Completeness and representativeness. Completeness means that the amount of information in the measure system is both necessary and sufficient, and a complete group of indicators is composed of several indicators. Therefore, in the design of evaluation index system should be done as little as possible, that is, with fewer indicators more accurately reflect the regional technological innovation ecosystem suitability of the whole picture. At the same time, on the basis of completeness, it is necessary to avoid the complicated index and select the comprehensive index and professional index with sufficient representative to express the coverage side accurately and concisely.

Comparability and operability principles. Because of the wide applicability of the regional ecosystem assessment index system for regional technological innovation, the comparability of the regional assessment indicators should be taken into account in the selection of indicators, the meaning of the assessment indicators, the statistical caliber and scope should be standardized as much as possible. At the same time, on the basis of accurately reflecting the suitability of the regional technological innovation ecosystem, try to select the common index of commonality and ensure the operability of the index system.

\section{Analysis on the Suitability of Regional Technological Innovation Ecosystem}

In the selection of empirical objects, we choose 16 provinces representing Chinese regional development as the object of analysis, namely Beijing, Tianjin, Liaoning, Shanghai, Zhejiang, Henan, Hunan, Guangdong, Chongqing, Sichuan, Shaanxi, Xinjiang, Hebei, Jiangsu, Shandong, Hubei and so on. The data from this study are mainly from two aspects: one from the statistical yearbook, including China Statistical Yearbook, China Science and Technology Statistical Yearbook and China Communications Statistical Annual Report; the other part comes from the National Bureau of Statistics official website. Which R \& D expenditure and large and medium-sized industrial enterprises output value of new products from the National Bureau of Statistics official website of the statistical report; communications investment in fixed assets from the 2005 China Communications Statistical annual report; the total number of scientific and technical personnel and research institutions from the Chinese science and technology statistics Yearbook 2006; other data are derived from China Statistical Yearbook 2007.

From the development trend of each province, Guangdong's technological innovation ecosystem has the highest degree of fitness, followed by Beijing, Jiangsu and Shanghai, and the lower scores are Hunan, Shaanxi, Chongqing and Xinjiang. This shows that from the current situation, Guangdong, Beijing, Jiangsu and Shanghai and other places relative to other areas in terms of more conducive to innovation and innovation activities to carry out the main. Xinjiang, Chongqing, Shaanxi, Hunan and other places of innovation is relatively weak, but also has a greater development and evolution of 
space. This is clearly demonstrated in the evolutionary momentum analysis of regional technological innovation ecosystem. The dynamic momentum of technological innovation ecosystem in Xinjiang, Chongqing, Shaanxi and Hunan is relatively large.

From the point of view of regional development, the suitability of technological innovation ecosystem in eastern China is relatively high, such as Guangdong, Shanghai, Jiangsu and Zhejiang provinces. The suitability of technological innovation ecosystem in the western region is relatively weak, such as Xinjiang, Shaanxi, Chongqing and other western provinces. In the central region, the degree of suitability of technological innovation ecosystem is in the middle position, such as Hubei, Henan, Hunan and other central provinces. This shows that from the current development trend of technological innovation ecosystem, Chinese regional technological innovation ecosystem suitability from east to west shows the status of the ladder.

From the comparison of the evaluation indexes of regional technological innovation ecosystem, Guangdong, Beijing, Shanghai, Jiangsu, Zhejiang and Shandong are in the best niche position in some indicators. For example, Guangdong occupies the optimal niche in terms of regional GDP, the number of patent licenses, the output value of new products of large and medium-sized industrial enterprises, etc. Beijing occupies the optimal niche in terms of technology market turnover, R \& D expenditure and so on. And the average wage to occupy the optimal niche; Zhejiang in the number of industrial enterprises, the total number of workers in colleges and universities in Jiangsu, Shandong, fixed investment in equipment, etc. occupy the best niche. And there is a certain gap between the real niche and the optimal niche in most provinces, which requires the provinces that do not reach the optimal niche. The provinces with the best niche should be the benchmarks to further improve the ecosystems suitability index of technological innovation To represent the environment, to enhance the suitability of technological innovation ecosystem.

Of course, the regional technological innovation ecosystem suitability values only reflect the overall development trend of regional innovation environment, and did not deny some of the specific areas of the region's innovative ecological environment. In fact, from the data collection and analysis process, we also found that in some areas where technological innovation ecosystems have low scores, there are also very good blocks of technological innovation ecosystems that are well- To promote the political, economic and legal environment for innovation activities to carry out innovative activities. For example, Xi'an Hi-tech Industrial development zone, economic and technological development zone, etc. It has a good demonstration role in becoming the leading regional innovation and development.

\section{Conclusion}

Through the empirical test of the evaluation model, we can see that the evaluation system of the regional ecosystem of technological innovation can better meet the goal of model construction, and can better reflect the basic situation of regional technological innovation ecosystem suitability, Analysis of the relevant indicators of regional ecosystems' suitability, so that decision makers can grasp the advantages and disadvantages of regional technological innovation ecosystem, and make better policy of regional innovation and technology policy. Through the empirical analysis of the suitability of regional technological innovation ecosystem in China, it can be found that the regional ecosystem of China is better than the central and western regions in the eastern region. At the same time, some concrete measures of the suitability of regional technological innovation ecosystem there are some imbalances in the distribution of the optimal niche, and there are some gaps between the real niche and the optimal niche in many regions, especially the gap between the central and western regions. This requires the decision-makers in the lower real-life areas to take full control of the status quo of regional technological innovation ecosystems, grasp the advantages and disadvantages of the region's own conditions, formulate good science and technology policies, and promote the continuity of regional technological innovation ecosystems healthy growth. 


\section{Acknowledgment}

2016 The School-local cooperation mode of electronic commerce to help Zizhou county economic development research"Funded by Xi'an International University

\section{References}

[1] Niu Haipeng, Zhao Tongqian, Zhang Anlu, Li Mingqiu. Study on sustainable utilization of cultivated land based on niche suitability [J] .Acta Ecologica Sinica, 10 (2009)

[2] Zhou Qing, Chen Guyong, Zhang Dinghua. An Empirical Study on the Suitability of Zhejiang Regional Technological Innovation [J]. Research and Development Management, 02 (2009)

[3] Wang Huanxiang, Sun Fei, Duan Xuemin. 30 years of reform and opening up Chinese regional innovation system evolution characteristics and dynamic analysis [J]. Science and Science and Technology Management, 12 (2008)

[4] Meng Deyou, Lu Yuqi. Study on Urban Niche Based on Niche Theory [J]. Journal of Regional Studies and Development, 02 (2008)

[5] Wang Hanhua, Liu Yanfang. Study on optimal allocation of land resources based on niche and constraint CA [J]. Chinese Journal of Population and Environment, 02 (2008)

[6] Li Zizhen, Han Xiaozhuo, Li Wenlong. Study on population evolution kinetics model with niche construction and its application [J]. Journal of Applied Mathematics and Mechanics, 03 (2006) 